\title{
The relative influence of pitch and timbre on the apparent location of sound in the median sagittal plane*
}

\author{
ROBERT A. BUTLER \\ Departments of Surgery (Otolaryngology) and Psychology, University of Chicago, Chicago, Illinois 60637
}

\begin{abstract}
Listeners were requested to locate sounds originating in the median sagittal plane (MSP). The stimuli, 75- $\mu$ sec pulses, were repeated at a rate of 200 times/sec and were filtered to transmit narrow bands centered about $0.63,1.6,2.5$, or $6.3 \mathrm{kHz}$. Despite the sameness of pitch generated by all stimuli, Os perceived the $0.63-, 1.6-$, and $2.5-\mathrm{kHz}-\mathrm{centered}$ sounds as originating low, middle, and high, respectively, in the MSP, regardless of their actual positions. The stimuli centered about $6.3 \mathrm{kHz}$, on the other hand, were located accurately by most Os. These findings were interpreted to mean that under conditions of inadequate auditory cues, timbre, not pitch, influences perceived elevation. The implication is that timbre also served as the cue for the apparent elevation of those sounds which, due to their high-frequency components, can be located accurately.
\end{abstract}

Despite the absence of binaural difference cues, auditory stimuli positioned vertically in the median sagittal plane (MSP) have spatial referents. If the sound is complex and includes the higher audio frequencies $(7-8 \mathrm{kHz})$, the location judgments of a normal hearing person correspond closely to the actual location of the sound source (Roffler \& Butler, 1968). If, on the other hand, tonal stimuli are presented, their apparent location no longer corresponds to their actual location. But, each still possesses a spatial referent upon which most listeners agree: high-frequency tones appear to originate above lower frequency tones. Undoubtedly this phenomenon has been recognized for decades, if not centuries: its experimental verification, however, is recent (Pratt, 1930; Trimble, 1934). In his brief and engaging discussion of the phenomenon, Pratt outlined the opposing interpretations. One, advanced by Stumpf, was that associative cues were responsible for the apparent location of tonal stimuli. In particular, the words "high" and "low," as applied to the pitch of a tone, influence strongly our location judgments. Pratt rejected this interpretation, contending that "... prior to any associative addition there exists in every tone an intrinsic spatial character which Yeads directly to the recognition of differences in height and depth along the pitch-continuum [p. 282]." Trimble (1934). in turn, questioned Pratt's explanation. He argued that if high-pitched sounds appear high in space and low-pitched sounds, low, irrespective of their actual locations, how can pitch serve as an effective cue for sound localization? Trimble believed that the spectral composition of a sound, or in his words, "differences in richness of partials," furnishes the cues for vertical location.

The present experiment resumes the argument started by Trimble. It was designed so that the fundamental

*This research was supported in part by NINDS Grant NS-03358 of the USPHS. The author gratefully acknowledges Neil Planert's assistance in testing the Ss. frequency (pitch) of the stimuli remained the same, but their spectral composition (timbre) changed. Three of the four experimental conditions were contrived to prevent the apparent location of the stimuli from corresponding consistently to their physical locations. This was accomplished simply by eliminating the higher frequencies $(>6.0 \mathrm{kHz}$ ) from the stimuli. For these conditions, the question was: What factor would determine location judgments-the pitch of the stimulus or its timbre? If pitch governs location judgments, all stimuli should appear to originate from the same region of the MSP, since all stimuli presumably had the same pitch. And, under the experimental conditions employed in this study, the sounds should be perceived as originating low in space, since the pitch of the stimuli was low. If timbre governs location judgments, the apparent elevation of the sounds should be distributed in various regions in the MSP, depending on the spectral composition of the respective stimuli. The remaining experimental condition provided information on how accurately this particular set of Os could locate sounds in the MSP when the stimulus cues were inadequate.

\section{METHOD}

Testing was conducted in a sound-treated room used previously for sound-localization studies. Its walls and ceiling are covered with acoustically absorbent material, except for an observation window at the rear of the room. Its floor is carpeted. Drapes suspended from the ceiling describe an arc extending from approximately $250 \mathrm{deg}$ through $0 \mathrm{deg}$ through $110 \mathrm{deg}$ with respect to the listener, who faces the center of the arc. Although this room is not anechoic, it is significant to note that sound localization data collected here are in accord with those of similar studies carried out by investigators working in anechoic chambers. Indeed, even the small, but consistent. biases in location judgments expressed as a function of stimulus frequency which were reported by Sandel et al (1955) have been replicated (Butler et al, 1967).

Straight ahead of the listener were five loudspeakers. arranged vertically in the MSP. Separated by $15 \mathrm{deg}$. center-to-center. the upper loudspeaker was positioned $30 \mathrm{deg}$ above and the lower loudspeaker $30 \mathrm{deg}$ below the listener's aural axis. Each 


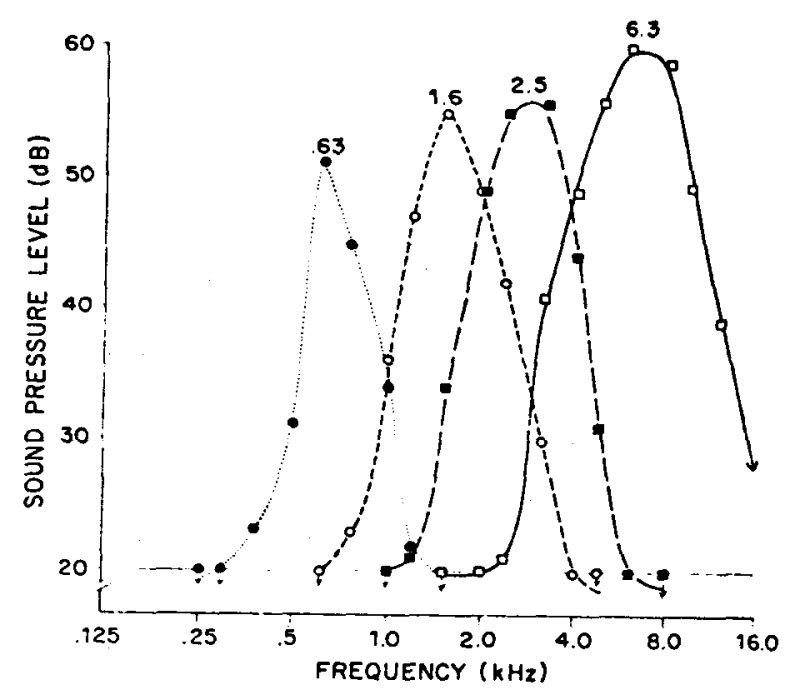

Fig. 1. Distribution of acoustic energy within one-third octave bands for the various stimuli employed in this study.

loudspeaker was designated by a number, and the listener's task was to identify that loudspeaker from which the sounds originated. Stimulus presentation continued until the choice was announced. Listeners were requested not to move their heads. A headrest was provided to promote compliance.

The sounds were on for $200 \mathrm{msec}$, then off for $200 \mathrm{msec}$. Stimulus rise-fall time was $10 \mathrm{msec}$. The important characteristic of the stimuli was their fine structure; the envelope of each contained $75-\mu \mathrm{sec}$ pulses repeated at a rate of $200 / \mathrm{sec}$. The pulses were delivered to two active, variable filters (SKL, Model 302), arranged in series and set to bandpass different sectors of the audio frequency range. Figure 1 describes the sound spectrum employed in each of the four experimental conditions. These data were recorded by means of a microphone placed in the space that would be occupied by the center of the listener's head during testing. The loudspeaker generating the stimuli was located at a height level with that of the aural axis of a would-be listener. The microphone's output was fed to an amplifier (Bruel \& Kjaer, Type 2603) and then to a filter (Bruel $\&$ Kjaer, Type 1602), where the acoustic energy of each stimulus was ascertained in one-third octave steps.

Twelve listeners, whose thresholds for 0.25 through $8.0 \mathrm{kHz}$ were within $15 \mathrm{~dB}$ of audiometric zero (ISO, 1964), participated. Each was given four test sessions. A test session consisted of 50 trials, or location judgments. The experimental conditions (differently filtered stimuli) were presented in a haphazard order; so was the order of loudspeaker activation. At the completion of an O's final test session, each loudspeaker had generated each of the four different stimuli an equal number of times, namely, 10. The loudness of the various stimuli was equated in the judgment of three experienced listeners by decreasing the overall intensity of the $6.3-\mathrm{kHz}$-centered stimulus by $2 \mathrm{~dB}$ and increasing the overall intensity of the $0.63-\mathrm{kHz}$-centered stimulus by $2 \mathrm{~dB}$ from the values shown in Fig. 1. To preclude a possible systematic influence of loudness differences among loudspeakers on location judgments, stimulus intensity generated by the various loudspeakers was sometimes increased and sometimes decreased by $5 \mathrm{~dB}$. The intensity changes followed a quasirandom order.

\section{RESULTS}

Figure 2 shows the distribution of location judgments for each of the four differently filtered pulses. For three of these conditions, the spectral composition of the sounds unquestionably influenced their apparent location. Stimuli whose spectral content centered about $0.63,1.6$, and $2.5 \mathrm{kHz}$ were judged to originate in the lower, middle, and higher regions of the MSP, respectively. Location judgments of stimuli whose spectral content centered about $6.3 \mathrm{kHz}$, on the other hand, were more evenly distributed among the five loudspeakers. The reason for this latter finding is apparent. Judged location coincided with actual location. The data, as organized in Fig. 3, demonstrate this point. Here, the distributions of error scores for the four experimental conditions are plotted. An error score of " 0 " indicates that the loudspeaker that generated the sound was correctly identified. An error score of " 1 " means that the listener judged the loudspeaker adjacent to the "correct" loudspeaker as the source of the sound. An error score of "2" or " 3 " means that the judged source of the sound was twice or thrice removed from the loudspeaker that actually generated the sound, etc. As can be seen, only the location judgments of the sound centered about $6.3 \mathrm{kHz}$ corresponded closely to the actual location of the sound source. Approximately $50 \%$ of all location judgments were correct; rarely was there an error greater than $15 \mathrm{deg}$, i.e., two or more loudspeakers removed from the correct one.

The pooled data, as shown in Figs. 2 and 3, provide an overall and reasonably accurate account of the facts. Yet, it is instructive to examine the data with regard to the individual's contribution. Without becoming encumbered by details, suffice it to say that the error score distribution observed for 10 of the 12 Os when locating stimuli centered about $6.3 \mathrm{kHz}$ differed significantly from chance $(p<.01 ;$ chi-square test $)$. This nonchance distribution can be attributed to accuracy in localization. One of the 2 Os who failed to perform proficiently was available for further testing. She was given a session in which only the stimuli peaking around

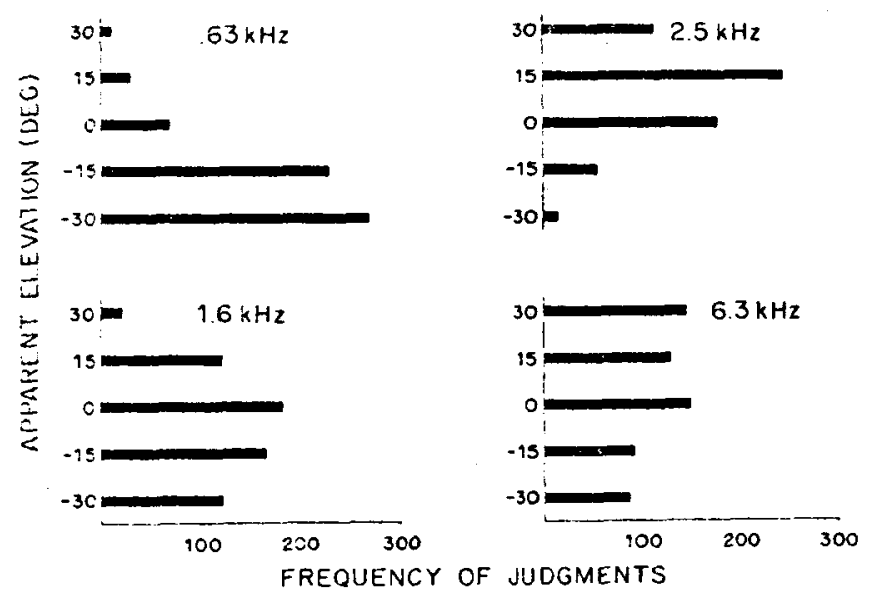

Fig. 2. Frequency distribution of location judgments for the differently filtered stimuli. 
$6.3 \mathrm{kHz}$ were presented. Each loudspeaker was activated on 10 trials, with the order of activation being quasirandom. Under these conditions, she performed excellently: 17 correct responses and 29 responses to that loudspeaker only once removed from the correct loudspeaker. Only 4 judgments were grossly incorrect. There were 6 other instances where error score distribution differed from chance expectancy beyond the .01 confidence level: 4 for the stimuli centered about $2.5 \mathrm{kHz}$ and 2 for the stimuli centered about $1.6 \mathrm{kHz}$. In all but one of these cases, the observed distribution could be traced to a propensity to perceive the stimuli as originating at 0 -deg elevation. This concentration of location judgments at or around the center of the vertical array of loudspeakers is the only other factor besides localization accuracy that can generate a nonchance distribution of error scores. For when a listener does this, she or he is unlikely to make a large error of judgment which, by chance, should be made occasionally.

\section{DISCUSSION}

The trend of the group data is unequivocal: Under conditions unfavorable for accurate localization in the MSP, the apparent elevation of a sound was governed by its spectral composition, not by its periodicity. The apparent height of the stimulus increased with increases in the frequency of the spectral composition. This relation held for all except the $6.3-\mathrm{kHz}$-centered stimulus. The spectral composition of this stimulus included frequencies that are known to be essential for accurate localization of vertically positioned sounds (Roffler \& Butler, 1968). Then, the location judgments of most Os were no longer illusory; they correlated with the physical location of the sound. The $6.3 \mathrm{kHz}-\mathrm{kenter}$ stimulus condition also provided information relevant to the attentiveness of the listeners. Specifically, had Os succumbed to a pattern of assigning location of sounds solely on the obvious differences in the frequency composition of the various stimuli, they would have assigned the $6.3-\mathrm{kHz}$-centered stimulus to the highest position in the MSP. Only 2 Os settled on this simple, but incorrect, solution to the localization task; 10 did not.

Whether the question that motivated this study (viz, Does timbre or pitch govern location judgments in the absence of adequate cues?) was answered can be debated. While the periodicity of all stimuli remained the same, some persons would, no doubt, insist that the pitch of the sounds was changed when their spectral composition was modified. However, investigators who have developed the area of "periodicity" pitch would maintain that such a statement reflects a confusion of pitch with timbre-a confusion which occurs frequently when naive listeners are used as Ss (Ritsma, 1970).

The present study articulates with Ritsma's work in yet other ways. He demonstrated that the frequency

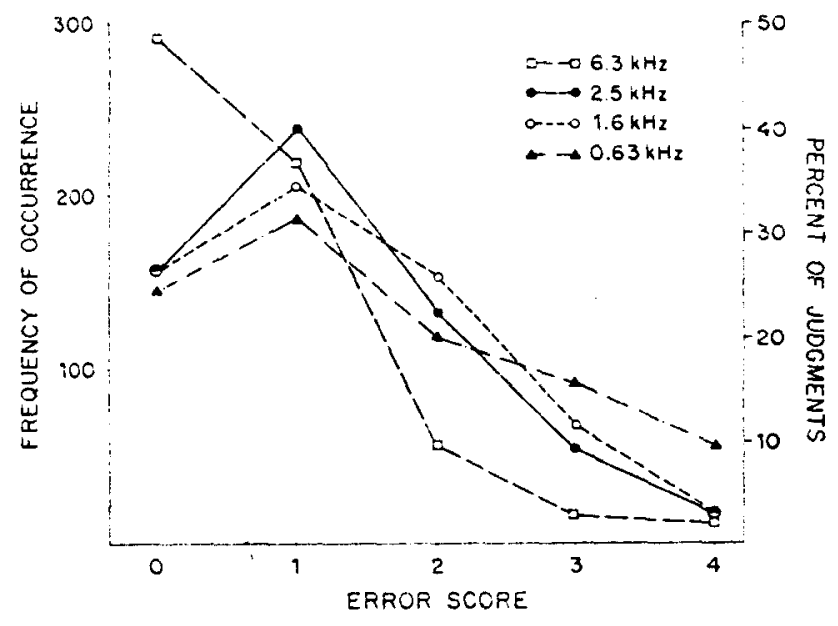

Fig. 3. Error score distribution expressed also as proportion of location judgments for the differently filtered stimuli.

band that provided the most dominant sensation of periodicity pitch was that in the region of the third, fourth, and fifth harmonic (Ritsma, 1967). This frequency band also produces the strongest sensation of repetition pitch (Ritsma \& Bilsen, 1970). Those stimuli, in the study reported here, that centered about $0.63 \mathrm{kHz}$ appeared to possess the richest tonality of pitch, and $0.63 \mathrm{kHz}$ is, of course, in the neighborhood of the third harmonic of the $0.2-\mathrm{kHz}$ fundamental. It should be emphasized, however, that the frequency band centered as high as $6.3 \mathrm{kHz}$ still retained periodicity pitch. This, too, would be predicted on the basis of Ritsma's (1963) experiments on the upper frequency limit of tonal residue.

Are these data relevant to the more important problem of how we locate accurately sounds in the MSP? Perhaps. If timbre influences apparent elevation when the auditory cues are inadequate, i.e., absence of high frequencies, it may do so under favorable listening conditions. Certainly, there are data which are consonant with this idea. The spectral composition of a sound changes in a systematic fashion within the frequency range of $7-10 \mathrm{kHz}$ as a sound source is moved from below to above the aural axis (Shaw \& Teranishi, 1968; Shaw, 1971). These spectral differences, which can be attributed largely to a transformation of the acoustic waveform by the pinna, are paralleled by differences in the timbre of a sound. Damaske (1971) has reviewed the work of several European investigators, all of which point to the importance of spectral cues as the basis for determining whether a sound is originating from in front, above, or behind. Moreover, by judicious use of filtering, it was shown that spectral composition could also provide location cues for horizontally positioned sounds, even when listening binaurally.

The extent to which we learn to associate timbre differences with differences in spectral composition resulting from changes in the position of a sound source, 
particularly in elevation, is not known. But one does have the compelling impression that the perception of elevation is immediate-no ratiocinations are involved.

\section{REFERENCES}

Butler, R. A., Roffler, S. K., \& Naunton, R. F. The role of stimulus frequency in the localization of sound in space. Journal of Auditory Research, 1967, 7, 169-180.

Damaske, P. Die psychologische Auswertung akustischer Phänomene. Seventh International Congress on Acoustics, Budapest, 1971, 21, G 2, 43-56.

ISO. See Davis, H., \& Kranz, F. W. The international standard reference zero for pure-tone audiometers and its relation to the evaluation of impairment of hearing. Journal of Speech \& Hearing Research, 1964, 7, 7-16.

Pratt, C. C. The spatial character of high and low tones. Journal of Experimental Psychology, 1930, 13, 278-285.

Ritsma, R. J. Existence region of the tonal residue II. Journal of the Acoustical Society of America, 1963, 35, 1241-1245.

Ritsma, R. J. Frequencies dominant in the perception of the pitch of complex sounds. Journal of the Acoustical Society of America, 1967, 42, 191-198.

Ritsma, R. J. Periodicity detection. In R. Plomp and G. F. Smoorenburg (Eds.), Frequency analysis and periodicity detection in hearing. Leiden: Sijthoff, 1970. Pp. 250-266.
Ritsma, R. J., \& Bilsen, F. A. Spectral regions dominant in the perception of repetition pitch. Acustica, 1970, 23, 334-339.

Roffler, S. K., \& Butler, R. A. Factors that influence the localization of sound in the vertical plane. Journal of the Acoustical Society of America, 1968, 43, 1255-1259.

Sandel, T. T., Teas, D. C., Feddersen, W. E., \& Jeffress, L. A. Localization of sound from single and paired sources. Journal of the Acoustical Society of America, 1955, 27, 842-852.

Shaw, E. A. G. Acoustic response of external ear with progressive wave source. Paper presented at the 82nd Meeting of the Acoustical Society of America, Denver, Colorado, 1971.

Shaw, E. A. G., \& Teranishi, R. Sound pressure generated in an external-ear replica and real human ears by a nearby point source. Journal of the Acoustical Society of America, 1968, 44, 240-249.

Trimble, O. S. Localization of sound in the anterior posterior and vertical dimensions of auditory space. British Journal of Psychology, 1934, 24, 320-334.

(Received for publication December 18, 1972; revision received April 10,1973.) 